\title{
Editorial
}

\section{Women in paediatrics}

In most countries women are relatively over represented in both the training and career grades of paediatrics when compared with other branches of medicine. This applies to those countries where paediatrics has lowly status and lowly pay, and also to those countries where paediatrics has a high status and is a well paid specialty. This means that most paediatric organisations should have the subject of women doctors high on their list of priorities.

The need for such urgent consideration is particularly necessary in the UK. In the last eight years there has been a big increase in the proportion of women admitted to medical schools; in most medical schools it is now between 35 and $50 \%$. Yet the present paediatric career structure and training, which evolved to suit the needs of male doctors (or unmarried women doctors), is unsatisfactory for those who wish to combine paediatric work with bringing up a family. At present those girls who entered medical school at the time of the doubling or trebling of the female intake are still either students or junior staff. Their most pressing needs will arise in a few years' time when they are competing for higher training and career posts, and when they themselves have children.

The problem for women doctors in the UK could be said to apply to all branches of medicine but since paediatrics has an excess of women we have a particular responsibility. There has been frequent mention of the plight of women doctors by national medical organisations. But national decisions are influenced most heavily by the specialties of adult medicine, surgery, obstetrics, and gynaecology. At present those specialties have comparatively few women in their ranks. The problems for these influential organisations will not be as pressing as those of certain other branches of medicine-such as paediatrics. Therefore the national associations of paediatrics should lead the investigation into the problems facing women doctors and suggest other solutions. Many will hope that they will be able to suggest realistic training and career posts for married women. If they cannot do so then it would seem that they have a duty to make it plain to junior women doctors that career prospects are bleak, and they should dissuade the present preponderance of women from entering paediatrics.

Democratic decisions reached by national medical associations about training and career grades may seem logical, but when applied to the differing needs within different branches of medicine they can become illogical and useless. That which may be helpful to one branch of medicine may not be helpful to another. Therefore any national organisation representing only one branch of medicine should suggest ways of providing satisfactory training and career posts for women, but should not imply that their solution is necessarily correct for all women doctors in other branches of medicine. They must simply put their own house in order and insist on the right to do so. 